\title{
Stability of Tissue Augmented with Deproteinized Bovine Bone Mineral Particles Associated with Implant Placement in Anterior Maxilla
}

\author{
David Buntoro Kamadjaja $\mathbb{D}^{1},{ }^{1,2}$ Ni Putu Mira Sumarta, ${ }^{1}$ and Andra Rizqiawan ${ }^{1}$ \\ ${ }^{1}$ Department of Oral and Maxillofacial Surgery, Faculty of Dental Medicine, Universitas Airlangga, Surabaya, Indonesia \\ ${ }^{2}$ Stem Cell Research and Development Center, Universitas Airlangga, Surabaya, Indonesia \\ Correspondence should be addressed to David Buntoro Kamadjaja; david-b-k@fkg.unair.ac.id
}

Received 24 February 2019; Revised 11 September 2019; Accepted 12 September 2019; Published 27 October 2019

Academic Editor: Luis M. J. Gutierrez

Copyright (C) 2019 David Buntoro Kamadjaja et al. This is an open access article distributed under the Creative Commons Attribution License, which permits unrestricted use, distribution, and reproduction in any medium, provided the original work is properly cited.

\begin{abstract}
Background. Implant placement in defective anterior maxilla poses a great challenge regarding functional and aesthetic outcomes. Therefore, it requires predictable alveolar ridge augmentation. Deproteinized bovine bone mineral (DBBM) particle has commonly been used for bone grafting. However, it is associated with low resorption rates which potentially compromise the outcome of horizontal augmentation in conjunction with implant placement. Aims. This study is aimed at evaluating the stability of tissue augmented with DBBM particle associated with implant placement in the anterior maxilla. Materials and Methods. The inclusive criteria consist of patients being treated with guided bone regeneration (GBR) incorporating the use of DBBM particles with either a simultaneous or staged approach. The parameters analyzed include the implant survival rate, post-GBR clinical stability based on tissue resorption level, and the tissue stability between simultaneous and staged approaches. Statistical analysis using Mann-Whitney test is performed with significance determined at $p$ value $<0.05$. Results. Seventeen patients with 23 implant placements satisfy the criteria for this study. Simultaneous approach is adopted in 18 (78.3\%) implants and a staged approach in $5(21.7 \%)$ implants. The implant survival rate is $100 \%$. The evaluation of horizontal tissue stability reveals a low resorption level in 19 (82.6\%) implants, while moderate and high resorption levels are found in 3 (13.0\%) and 1 (4.3\%) implants, respectively. The statistical analysis shows that the simultaneous approach produces significantly $(p=0.005)$ lower resorption level compared to the staged approach. Conclusion. Horizontal ridge augmentation using DBBM particles associated with implant placement in the anterior maxilla produces good clinical stability. The stability appears to be higher in the simultaneous approach compared to the staged approach.
\end{abstract}

\section{Introduction}

Nowadays, increasing numbers of patients are seeking dental implant treatment due to its high success rate which poses significant challenges to clinicians dealing with such treatment. One such challenge in implant placement is tissue deficiency in the anterior maxilla leading to functional, structural, and aesthetic compromises which require horizontal ridge augmentation $[1,2]$.

Guided bone regeneration technique has been widely employed in horizontal augmentation [3] for which autogenous bone graft has represented the gold standard graft material. However, autogenous bone graft has been associated with limited donor sources and donor site morbidity [4]. Therefore, bone substitutes are now preferred since they permit minimally invasive surgery.

Deproteinized bovine bone mineral (DBBM) particle is a natural bone substitute with good osteoconductive properties which has been shown to achieve impressive results in various experimental and clinical studies [5-8]. However, DBBM has also been associated with very low degradation rates with the result that defect healing is characterized by graft particles being integrated within the new bone, rather than by complete replacement with new bone or bone regeneration 
[9]. This clinical study attempts to evaluate the stability of tissue augmented with DBBM particle in conjunction with implant placement in the anterior maxilla.

\section{Subjects and Methods}

This retrospective study evaluates patients treated with bone grafting procedures in conjunction with implant placement at the Outpatient Clinic, Dental Hospital, Universitas Airlangga, Surabaya, between July 2010 and August 2018. The study is certified to be ethically cleared by Health Research Ethical Clearance, Faculty of Dental Medicine, Universitas Airlangga (number 288/HRECC. FODM/VIII/2018). The inclusion criteria for the study are as follows. Patients present with reduced horizontal dimension of the alveolar ridge after extraction of the anterior maxillary teeth requiring implant replacement (Figure 1(a)). The augmentation procedure for alveolar defects consists of guided bone regeneration using DBBM particles and resorbable membrane through either a simultaneous approach in which the bone grafting procedure and implant placements are performed at the same time or a staged approach in which the bone grafting procedure is conducted prior to implant placement. Excluded from this study are patients with poorly controlled diabetes mellitus who fail to return for review or whose cases involve the application of bone graft other than DBBM. Informed consent forms are signed by all patients regarding the use of bone substitutes and implant placement.

Data collected during this study includes the distribution of sample sex and age, the duration of treatment in the simultaneous and staged approaches, the length of observation, and the distribution of the number of implant placement related with augmentation approaches. The parameters analyzed comprise the implant survival rate and the stability of alveolar tissue width post augmentation procedure involving the use of resorption level. The tissue resorption is established by measuring clinically the difference in alveolar width between the immediate post augmentation procedure (Figure 1(b)) and the follow-up at the end of the study period (Figure 1(c)). The data collected is then categorized into such scores as low if the difference is less than $1 \mathrm{~mm}$, moderate $=1-2 \mathrm{~mm}$, and high $=$ more than $2 \mathrm{~mm}$.

The surgical procedure within the simultaneous approach uses trapezoidal incision and mucoperiosteal flap in order to expose the defective bone. Bone preparation is performed in a slightly palatal direction as a means of achieving initial implant stability. An osseointegrated implant (Axiom ${ }^{\circledR}$, Anthogyr, France) is inserted until an initial stability of $35 \mathrm{Ncm}$ torque is achieved leaving implant threads exposed, at least, labially (Figure 2(a)). The peri-implant gap is filled and the horizontal bone defect augmented with DBBM particle (Cerabone ${ }^{\circledR}$, Botiss GmbH, Germany) (Figure 2(b)). A resorbable membrane (Jason Membrane ${ }^{\circledR}$, Botiss biomaterials $\mathrm{GmbH}$, Germany) is then placed over the bone graft particle. Healing abutments are installed in the immediate loading, while a cover screw is placed in the delayed loading. Primary closure is facilitated with a periosteal releasing incision. In the staged approach, the bone defects are filled with bone substitute particle, covered with membrane and primarily closed. The graft is allowed to heal for at least 6 months before implant placement is conducted.

In immediate loading cases, implant impression is undertaken approximately 2 months after implant placement. In delayed loading cases, phase 2 is initiated around 6 months post implant placement. Crestal incision is made to expose the implant platform and insert the healing abutment (Figure 2(c)). Approximately one month thereafter, when the soft tissue remodelling around the healing abutment is completed (Figure 2(d)), implant impression is undertaken with closed tray technique for final restoration (Figure 2(e)).

\section{Results}

A total of 17 patients with 23 dental implant placement fulfills the inclusion criteria outlined above, consisting of 9 females and 8 males with a mean age of 43.6 years (range 18 to 66 ). The length of observation ranged from 8 to 90 months (average 34.9 months). The augmentation was done with simultaneous approach in 18 (78.3\%) implant placements and with the staged approach in 5 (21.7\%) implant placements (Table 1). Complication documented is wound dehiscence and graft particle exposure in a case treated with a staged approach. A second operation is performed in order to remove the old graft and close the wound without compromising the remainder of the grafted site. The average treatment length in the simultaneous approach was 6.6 months compared to 14.5 months in the staged approach.

The implant survival rate was $100 \%$ as no failures are documented during the observation period. Direct horizontal tissue gain is clinically achieved after the augmentation procedure in all cases. The evaluation of clinical alveolar width (horizontal) stability shows low resorption level in 19 $(82.6 \%)$ implant placements, while moderate and high resorption levels are found in $3(13.0 \%)$ and 1 (4.3\%) implant placements, respectively (Table 2 ).

The evaluation of clinical horizontal tissue stability after GBR procedure using DBBM particle reveals that low resorption level is documented in $94.4 \%$ of implant placements augmented with simultaneous approach while moderate resorption level is found in 5.6\% implants and none is associated with high resorption level. On the contrary, low and moderate resorption levels are documented both in $40 \%$ of implants and $20 \%$ of them is related with high resorption level when augmented with the staged approach (Figure 3). Mann-Whitney test result confirms that the resorption level in the simultaneous approach is significantly $(p=0.005)$ lower compared to that in the staged approach.

\section{Discussion}

Implant treatment in the anterior maxilla or aesthetic zone should be performed taking into account one important consideration, i.e., the aesthetic expectations of the patient [10]. One of the major problems associated with early or delayed implant placement in the anterior maxilla is horizontal tissue deficiency due to resorption 


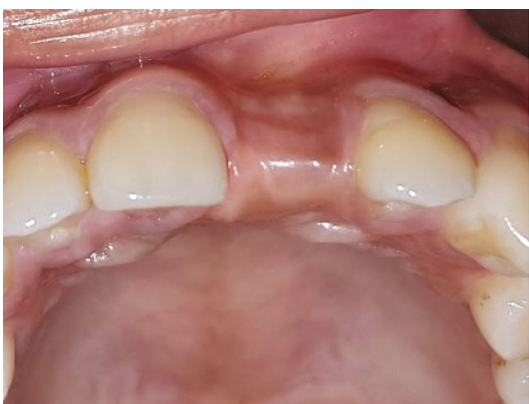

(a)

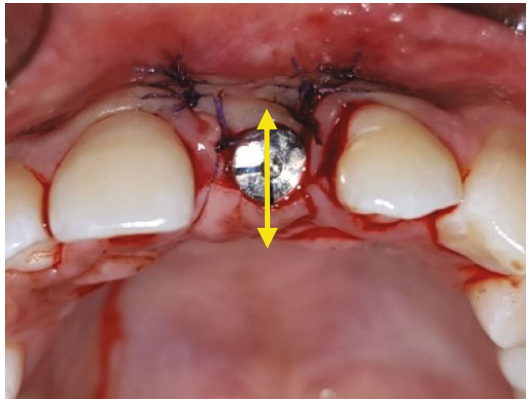

(b)

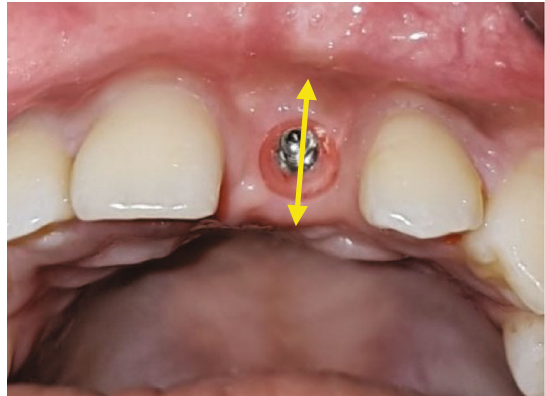

(c)

Figure 1: The measurement of augmented tissue resorption. (a) Postextraction horizontal defect of 21 alveolar ridge prior to augmentation; the tissue resorption is established by measuring clinically the difference in alveolar width between the immediate post augmentation procedure (b) and the follow-up at the end of the study period (c). The data collected is subsequently categorized into tissue resorption level as low if horizontal loss $<1 \mathrm{~mm}$, moderate $=1-2 \mathrm{~mm}$, and high $>2 \mathrm{~mm}$.

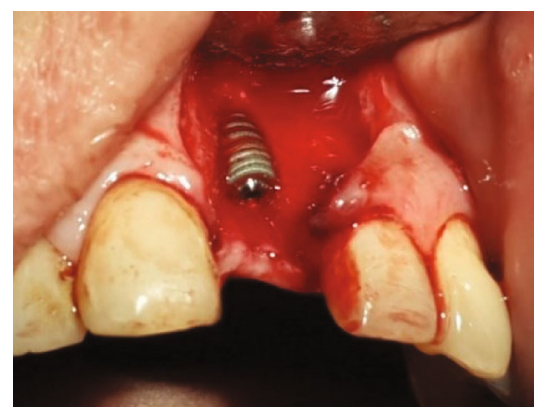

(a)

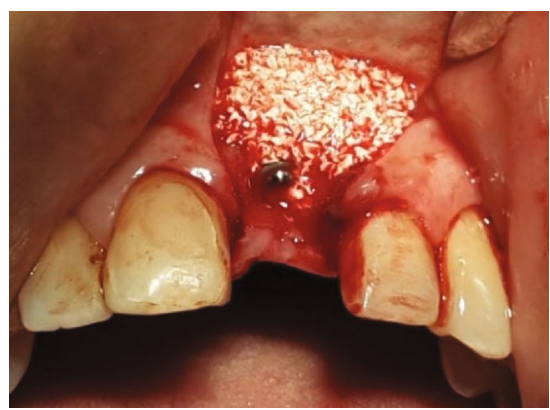

(b)

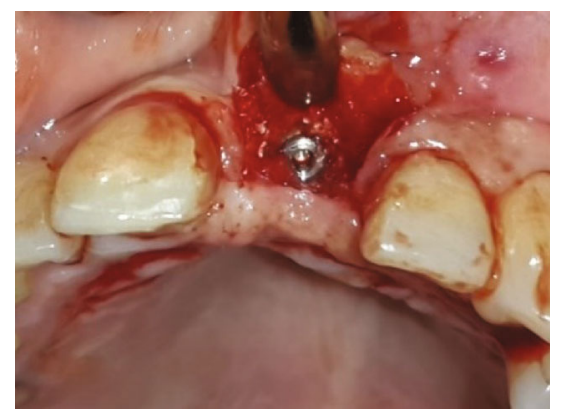

(c)

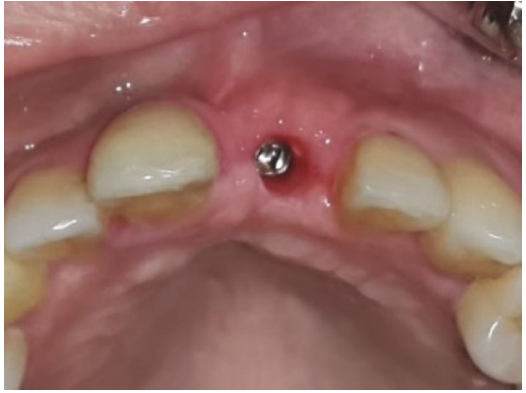

(d)

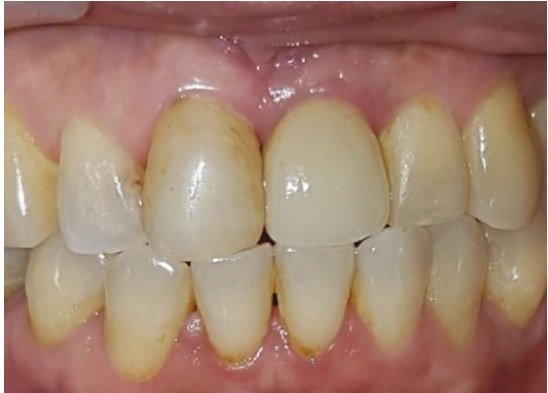

(e)

FIgURE 2: Simultaneous implant placement and horizontal augmentation in the anterior maxilla with delayed loading strategy. (a) Postextraction horizontal defect of 21 alveolar ridge, in which implant placement leaves two-thirds of the implant length exposed; (b) DBBM particle is applied to cover the implant and augment the alveolar defect; (c) at reentry six months after bone grafting, the augmented area is stable with still visible DBBM particles blended with the newly formed bone; (d) at prosthetic phase, the labial tissue width is shown to be comparable to the adjacent tissue; (e) final restoration shows healthy labial and interdental peri-implant tissue.

TABLE 1: The distribution of implant placement associated with simultaneous and staged augmentation approaches.

\begin{tabular}{lcc}
\hline & Simultaneous augmentation & Staged augmentation \\
\hline Male & 9 & 2 \\
Female & 9 & 3 \\
Total & $\mathbf{1 8}(78.3 \%)$ & $\mathbf{5}(21.7 \%)$ \\
\hline
\end{tabular}

of alveolar bone especially in the labial aspect. In order to achieve optimal peri-implant tissue support in the anterior maxilla, one should perform bone augmentation with bone graft or bone substitute to provide appropriate stability [11].

The results of this study showed that implant placements grafted with GBR using DBBM produce clinical stability in the horizontal dimension as the majority (82.6\%) exhibits low resorption levels during the follow-up review (Table 2). This result is consistent with those of previous studies which reveal good osteoconductivity and stability of the grafted bone [6,7]. This finding is most likely caused by the high volume stability of DBBM granules which may be associated with its poor degradability. Various studies have demonstrated that DBBM has a very slow degradation 
TABLE 2: The distribution of tissue resorption level after implant placement associated with simultaneous and staged approaches.

\begin{tabular}{lccc}
\hline $\begin{array}{l}\text { Resorption } \\
\text { level }\end{array}$ & $\begin{array}{c}\text { Simultaneous } \\
\text { augmentation }\end{array}$ & $\begin{array}{c}\text { Staged } \\
\text { augmentation }\end{array}$ & Total \\
\hline Low & 17 & 2 & $\mathbf{1 9}(82.6 \%)$ \\
Moderate & 1 & 2 & $\mathbf{3}(13.0 \%)$ \\
High & 0 & 1 & $\mathbf{1}(4.3 \%)$ \\
Total & $\mathbf{1 8}$ & $\mathbf{5}$ & $\mathbf{2 3}$ \\
\hline
\end{tabular}

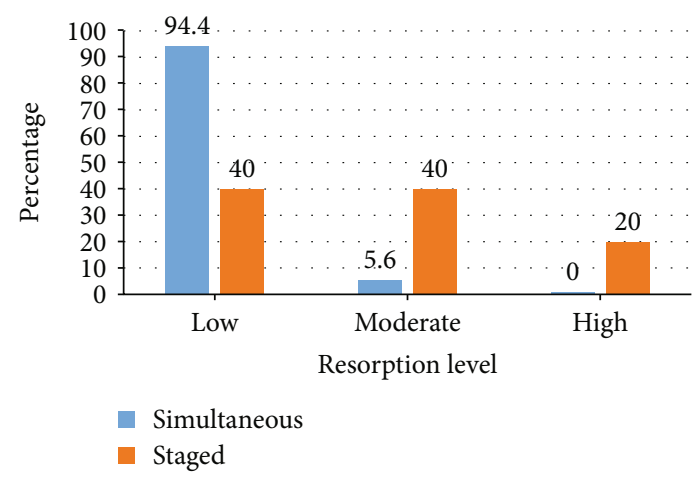

FIgure 3: The resorption level of simultaneous and staged augmentation with DBBM particle. The majority (94.4\%) of implant augmented with the simultaneous approach shows low resorption level, while only $5.6 \%$ is moderate and none is high level. On the other hand, with the staged approach, low resorption level is found in only $40 \%$ of implants, while the remainders are moderate (40\%) and high (20\%) levels. Mann-Whitney test result reveals significant difference $(p=0.005)$ in tissue resorption level between the two approaches.

rate, up to 3 to 4 years, or may not be completely degradable $[12,13]$. Moreover, its stability as graft material can last up to 1.5 years in the absence of dental implants [14].

Alveolar ridge augmentation can be performed at different time points during treatment and is generally categorized as staged or simultaneous approach. The simultaneous approach is obviously the technique preferred by both patients and clinicians since it reduces both the time and cost of treatment [15]. This finding is in accordance with the results of our study in which the treatment duration of the staged approach is more than twice than that of the simultaneous approach.

The result of this study reveals that GBR with the simultaneous approach produces significantly lower resorption level compared to the staged approach. Furthermore, moderate and high resorption levels are predominantly found in the staged approach (Figure 3). This result is noteworthy since DBBM is supposed to be mechanically stable. One plausible explanation would be that the staged augmentation approach is applied to cases with larger tissue deficit which precludes simultaneous implant placement. Larger deficit may have caused a certain degree of instability of the graft particles due to mucosal pressure or mechanical load exerted by provisional prosthesis or mastication [10] resulting in reduced horizontal dimension during the follow-up period.
Moreover, the second surgery performed for implant placement in the staged approach might have caused a certain degree of inflammation subsequently inducing bone resorption. These findings highlight the advantages of the simultaneous approach, namely, long-term stability of the grafted tissue compared to that of the staged approach.

The sole use of DBBM to promote augmentation of implant dehiscence might, theoretically, reduce the amount of bone regeneration around the exposed implant surface, thereby potentially compromising implant osseointegration. The DBBM particulate used in this study was made from bovine cancellous bone which demonstrates much higher porosity compared to cortical bone granules. The higher the porosity, the more effective the osteoconductivity of the graft material since it promotes faster bone formation around the exposed implant surface [16]. This is supported by the clinical findings in delayed-loading cases in this study in which good integration between DBBM particle and the new regenerated bone is evident (Figure 2). This suggests that the DBBM particle promotes osteoconduction supporting secondary implant stability during graft healing.

The novel method to measure tissue resorption level employed in this study is a relatively simple means of measuring the stability of grafted tissue. The parameter used in this method is purely based on clinical dimensions not taking into account the magnitude of grafted bone. Furthermore, the sample size used in this study is relatively small and considered heterogeneous to be able to obtain reliably strong inference. Further study is, therefore, required with bigger sample size and with imaging tools enabled to evaluate the magnitude of the grafted bone.

From the results of the study, it may be concluded that horizontal bone augmentation with deproteinized bovine bone mineral particle produces a clinically stable tissue in the anterior maxilla. Stability seems to be higher in the simultaneous approach compared to the staged approach.

\section{Additional Points}

Key Messages. Deproteinized bovine cancellous bone mineral particle represents a suitable bone substitute for guided bone regeneration of implant placement in the anterior maxilla due to its strong osteoconductive properties and structural stability. These allow for effective bone integration and stable grafted tissue leading to the effective functioning and aesthetic appeal of implant-supported prosthesis.

\section{Conflicts of Interest}

The authors declare that they have no conflicts of interest.

\section{Acknowledgments}

The author would like to thank the Faculty of Dental Medicine, Universitas Airlangga, and Dental Hospital, Universitas Airlangga, which has supported the study. The study would not have been possible without the permission from them. 


\section{References}

[1] D. C. Tong, K. Rioux, M. Drangsholt, and O. R. Beirnem, "A review of survival rates for implants placed in grafted maxillary sinuses using meta-analysis," The International Journal of Oral \& Maxillofacial Implants, vol. 13, pp. 175-182, 1998.

[2] H. L. Wang and K. Al-Shammari, "HVC ridge deficiency classification: a therapeutically oriented classification," The International Journal of Periodontics \& Restorative Dentistry, vol. 22, pp. 335-343, 2002.

[3] B. S. McAllister and K. Haghighat, "Bone augmentation techniques," Journal of Periodontology, vol. 78, no. 3, pp. 377-396, 2007.

[4] G. M. Raghoebar, C. Louwerse, W. W. I. Kalk, and A. Vissink, "Morbidity of chin bone harvesting," Clinical Oral Implants Research, vol. 12, no. 5, pp. 503-507, 2011.

[5] M. Caneva, D. Botticelli, E. Stellini, S. L. S. Souza, A. Salata, and N. P. Lang, "Magnesium-enriched hydroxyapatite at immediate implants: a histomorphometric study in dogs," Clinical Oral Implants Research, vol. 22, no. 5, pp. 512-517, 2011.

[6] M. Caneva, D. Botticelli, F. Pantani, G. M. Baffone, I. G. Rangel Jr., and N. P. Lang, "Deproteinized bovine bone mineral in marginal defects at implants installed immediately into extraction sockets: an experimental study in dogs," Clinical Oral Implants Research, vol. 23, no. 1, pp. 106-112, 2012.

[7] A. Barone, M. Ricci, J. L. Calvo-Guirado, and U. Covani, "Retracted: bone remodelling after regenerative procedures around implants placed in fresh extraction sockets: an experimental study in Beagle dogs," Clinical Oral Implants Research, vol. 22, no. 10, pp. 1131-1137, 2011.

[8] J. Nart, L. Barallat, D. Jimenez et al., "Radiographic and histological evaluation of deproteinized bovine bone mineral vs. deproteinized bovine bone mineral with $10 \%$ collagen in ridge preservation. A randomized controlled clinical trial," Clinical Oral Implants Research, vol. 28, no. 7, pp. 840-848, 2017.

[9] N. Venkataraman, S. Bansal, P. Bansal, and S. Narayan, "Dynamics of bone graft healing around implants," Journal of the International Clinical Dental Research Organization, vol. 7, no. 3, pp. 40-47, 2015.

[10] U. Kuchler and T. von Arx, "Horizontal ridge augmentation in conjunction with or prior to implant placement in the anterior maxilla: a systematic review," The International Journal of Oral \& Maxillofacial Implants, vol. 29, Supplement, pp. 1424, 2014.

[11] P. L. Santos, J. L. Gulinelli, C. S. Telles et al., "Bone substitutes for peri-implant defects of postextraction implants," International Journal of Biomaterials, vol. 2013, Article ID 307136, 7 pages, 2013.

[12] T. Berglundh and J. Lindhe, "Healing around implants placed in bone defects treated with Bio-Oss ${ }^{\circledR}$ : an experimental study in the dog," Clinical Oral Implants Research, vol. 8, no. 2, pp. 117-124, 1997.

[13] A. Skoglund, P. Hising, and C. Young, "A clinical and histologic examination in humans of the osseous response to implanted natural bone mineral," International Journal of Oral \& Maxillofacial Implants, vol. 12, pp. 194-199, 1997.

[14] B. McAllister, M. Margolin, A. Cogan, D. Buck, J. Hollinger, and S. Lynch, "Eighteen-month radiographic and histologic evaluation of sinus grafting with anorganic bovine bone in the chimpanzee," International Journal of Oral \& Maxillofacial Implants, vol. 14, no. 3, pp. 361-368, 1999.
[15] P. Fairbairn and M. Leventis, "Protocol for bone augmentation with simultaneous early implant placement: a retrospective multicenter clinical study," International Journal of Dentistry, vol. 2015, Article ID 589135, 8 pages, 2015.

[16] A. Scarano, "Maxillary sinus augmentation with decellularized bovine compact particles: a radiological, clinical, and histologic report of 4 cases," BioMed Research International, vol. 2017, Article ID 2594670, 6 pages, 2017. 


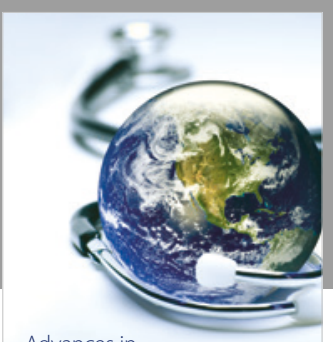

Advances in
Public Health

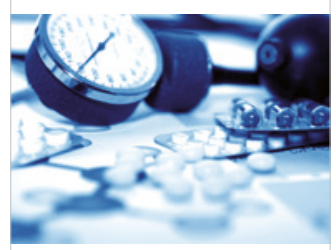

Case Reports in

Medicine

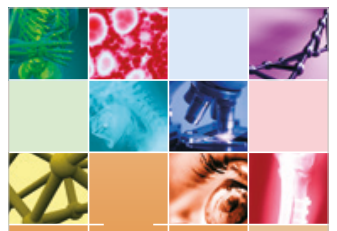

niernational Journal of

Biomaterials
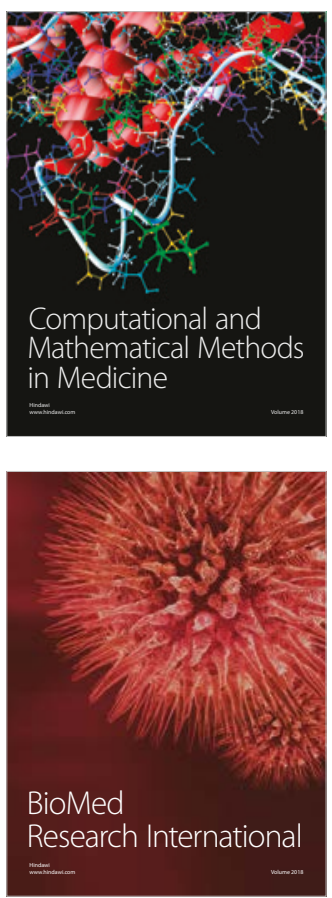

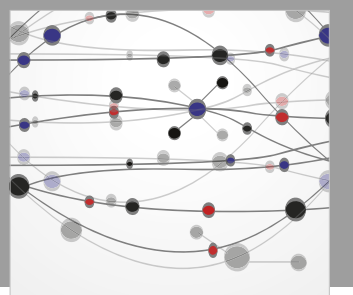

The Scientific World Journal Dentistry

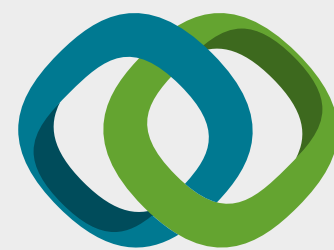

Hindawi

Submit your manuscripts at

www.hindawi.com
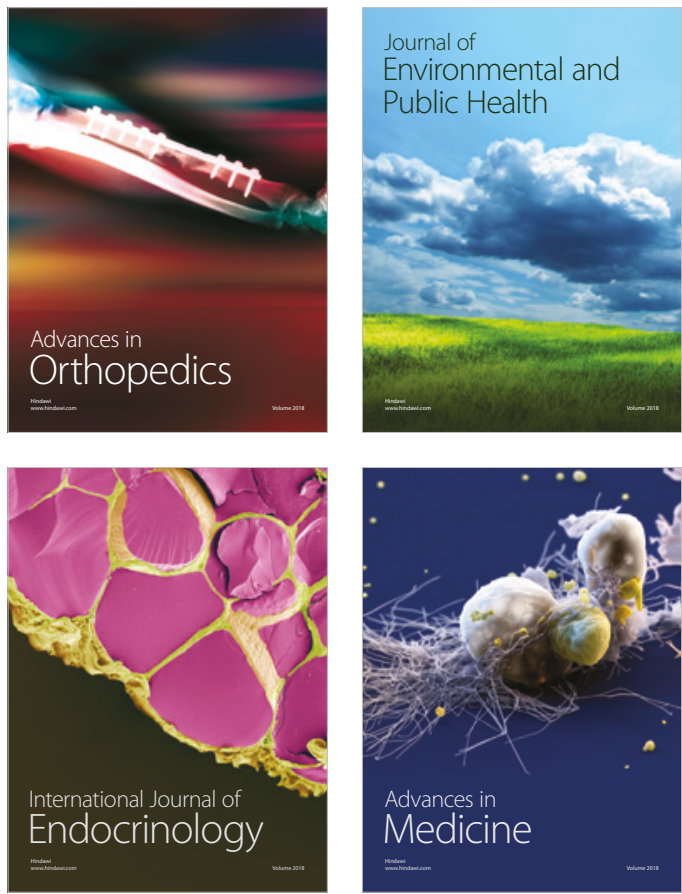
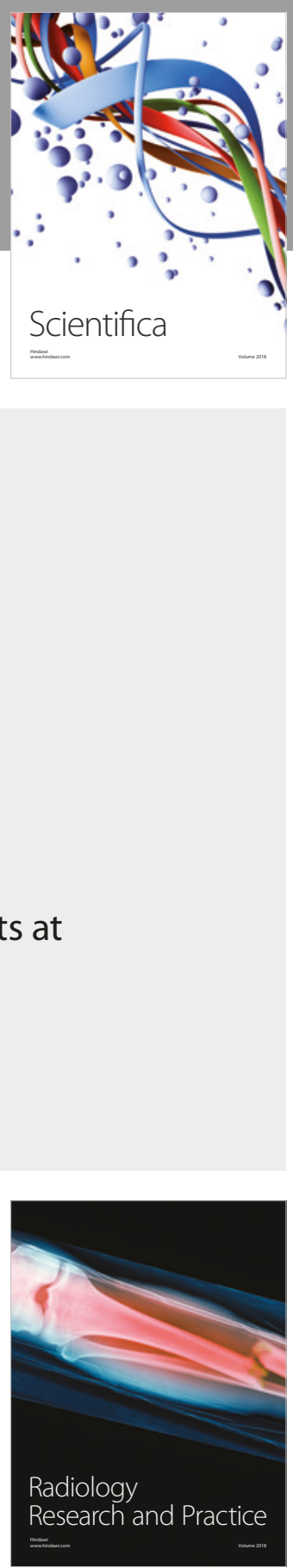

Scientifica

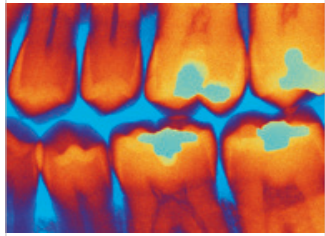

Case Reports in

Dentistry
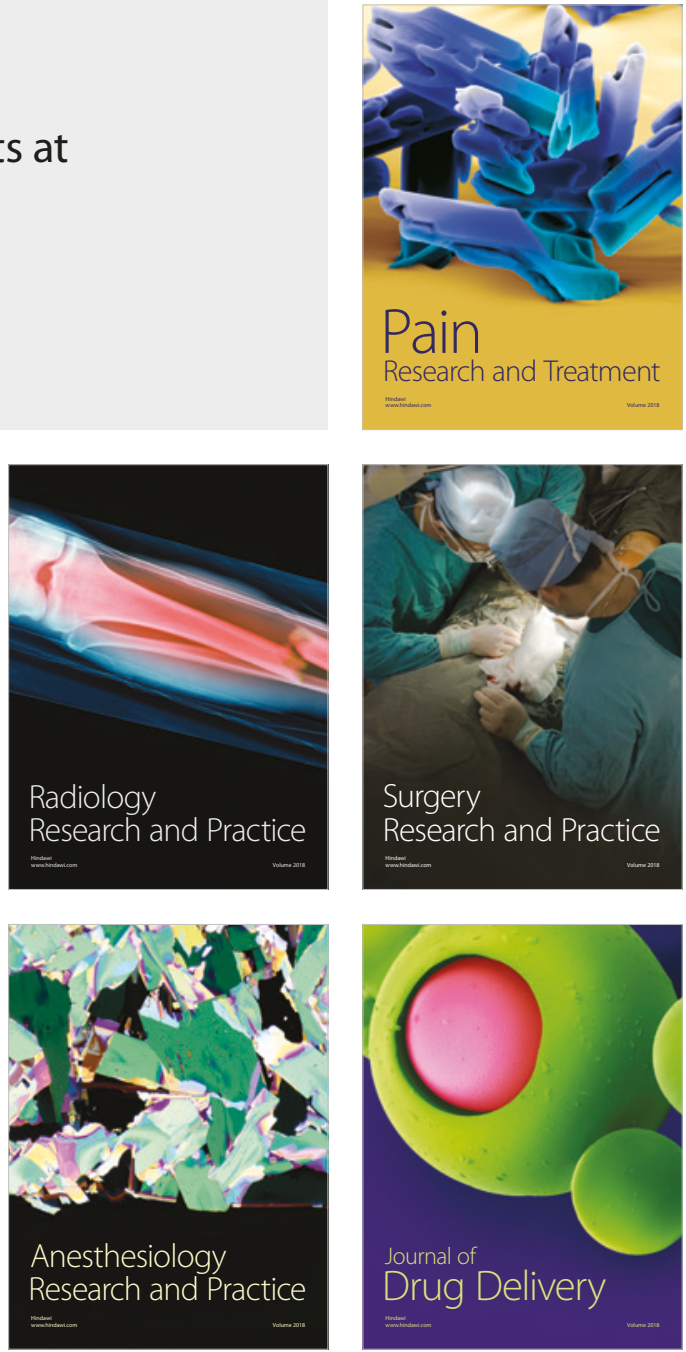